\title{
Cycloastragenol: An exciting novel candidate for age-associated diseases (Review)
}

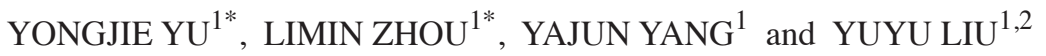 \\ ${ }^{1}$ Department of Pharmacology; ${ }^{2}$ Guangdong Key Laboratory for Research and Development of Natural Drugs, \\ Guangdong Medical University, Zhanjiang, Guangdong 524023, P.R. China
}

Received February 15, 2018; Accepted June 25, 2018

DOI: $10.3892 /$ etm.2018.6501

\begin{abstract}
Cycloastragenol (CAG) is a triterpenoid saponin compound and a hydrolysis product of the main active ingredient in Astragalus membranaceus (Fisch.) Bunge. An increasing body of evidence has indicated that CAG has a wide spectrum of pharmacological functions, which are attracting attention in the research community. The aim of the present review paper was to review and elucidate the advanced study of CAG. The focus was on advanced studies of CAG in English and Chinese databases; the literature was collected and reviewed to summarize the latest efficacy, pharmacokinetics and adverse reactions of CAG. Extensive pharmacological effects have been attributed to $\mathrm{CAG}$, including telomerase activation, telomere elongation,
\end{abstract}

Correspondence to: Dr Yuyu Liu, Department of Pharmacology, Guangdong Medical University, 2 Wenming Road, Zhanjiang, Guangdong 524023, P.R. China

E-mail: liuyuyu77@163.com

*Contributed equally

Abbreviations: CAG, cycloastragenol; TCM, traditional Chinese medicine; TERT, catalytic reverse transcriptase enzymes; TERC, telomerase RNA component; IPE, inositol trisphosphate; cAMP, cyclic adenosine monophosphate; Akt, protein kinase B; c-Src, proto-oncogene tyrosine-protein kinase; ERK, extracellular signal-regulated kinase; MEK, ERK kinase; JAK2, Janus kinase 2; STAT5b, signal transducer and activator of transcription 5b; HIV, human immunodeficiency virus; IPF, idiopathic pulmonary fibrosis; AIDS, acquired immunodeficiency syndrome; AG, astragaloside IV; CCI, cyclocephaloside I; CCE, cyclocanthoside E; FXR, Farnesoid X receptor; NAFLD, non-alcoholic fatty liver disease; MCD, methionine- and choline-deficient L-amino acid diet; NASH, non-alcoholic steatohepatitis; ROS, reactive oxygen species; T-AOC, total antioxidant capacity; T-SOD, total superoxide dismutase; HYP, hydroxyproline; MDA, malondialdehyde; ER, endoplasmic reticulum; IRE1, inositol-requiring enzyme 1; TXNIP, thioredoxin-interacting protein; NLRP3, NLR family pyrin domain containing 3; AMPK, 5'AMP-activated protein kinase; BMD, bone mineral density; NOAEL, no-observed-adverse-effect level; UGT, UDP-glucuronosyltransferase; GRAS, generally recognized as safe

Key words: Astragalus membranaceus (Fisch.) Bunge, cycloastragenol, telomerase activator anti-inflammatory and anti-oxidative properties; CAG has also been reported to improve lipid metabolism. Clinical research has demonstrated that CAG activates telomerase in humans and ameliorates various biomarkers. CAG is absorbed through the intestinal epithelium via passive diffusion and undergoes first-pass hepatic metabolism. Within a certain dose range, oral CAG is relatively safe; however, underlying mechanisms associated with CAG are not clear, and thus, we should be aware of potential adverse reactions associated with CAG. According to existing studies and clinical trials, CAG is safe and has broad application prospects. However, further studies are required to fully understand its efficacy and potential adverse reactions, and to ensure the proper use of CAG is applied to treat diseases clinically.

\section{Contents}

1. Introduction

2. Pharmacodynamics

3. Adverse reactions

4. Pharmacokinetics

5. Discussion

6. Conclusions and prospective

\section{Introduction}

Astragalus membranaceus (Fisch.). Bunge is a plant used in traditional Chinese medicine (TCM), with known ' $Q \mathrm{i}$ tonifying' adaptogenic effects, as documented in the Chinese Materia Medica. Astragalus membranaceus (Fisch.) Bunge has been used in TCM for over 2,000 years and is still being use in various herbal preparations. It is known to enhance immune functions; protect the liver; act as a diuretics; and have anti-aging, anti-stress, antihypertensive, and extensive antibacterial properties (1). Astragaloside IV (AG) is the main compound found in Astragalus membranaceus (Fisch.) Bunge while cycloastragenol (CAG), an algycone of AG, is a triterpenoid saponin compound obtained from AG hydrolysis products (Fig. 1) (2).

The Geron Corporation in cooperation with The Hong Kong University of Science and Technology screened natural compounds from Astragalus membranaceus extracts. CAG 
was identified as an anti-aging compound that promotes telomerase activity and wound closure (3). CAG is currently the only compound known to activate telomerase in humans (4), making it a great prospect for development. This study reviews the efficacy, pharmacokinetics studies, and adverse reactions of CAG, and provides a basis for further study into CAG.

\section{Pharmacodynamics}

\section{Efficacy}

\section{Efficacy base on pre-clinical studies}

Telomerase activation. Telomeres are located at the ends of linear chromosomes capped by nucleoprotein structures that consist of tandem repeats of hexameric sequences (TTAGGG in vertebrates) bound by a dedicated set of proteins. Telomeres shorten with every mitotic event. Telomerase is a ribonucleoprotein complex that lengthen telomeres and fundamentally consists of catalytic reverse transcriptase enzymes (TERT) and a telomerase RNA component (TERC). A key function of telomeres is to protect chromosomal ends from fusion and degradation by capping chromosomal ends. Cells recognize critically short telomeres as DNA damage; therefore, the shortest telomere, rather than average telomere length, is critical for cell viability and chromosomal stability (5). CAG activates telomerase, lengthens telomeres, and exerts a variety of pharmacological effects as introduced below (Fig. 2).

Telomerase activation and lengthening of telomeres. Several studies have shown that CAG activates telomerase both in vitro and in vivo. CAG activates telomerase and lengthens telomeres in a telomerase-dependent way in vitro; therefore, CAG decreases the percentage of critically short telomeres and DNA damage in the cell. CAG could activate telomerase in various cell types, including hematopoietic progenitors and mouse embryonic fibroblast haplo-insufficient for the TERC (MEF Terc ${ }^{+/-}$) cells. In vivo, dietary supplementation of CAG increases TERT expression in tissues such as bone marrow, lungs, heart, brain, and liver. Further, CAG rescued short telomeres in 1- and 2-year old female mice. However, data have shown that dietary supplementation with CAG does not impact the mean or maximum longevity of female mice $(6,7)$. Therefore, a necessary relation between telomerase activity and longevity does not likely exist.

The above study led to another question: How does CAG activate telomerase? Telomerase activity in human embryonic kidney HEK293 fibroblasts increases upon treatment with CAG and is not meditated via common secondary messenger pathways, including $\mathrm{Ca}^{2+}$, inositol trisphosphate (IP3), cyclic adenosine monophosphate (cAMP), and protein kinase B (Akt). However, CAG induced the phosphorylation of extracellular signal-regulated kinase (ERK) in many cell lines, including HEK 293 and HEK-neo keratinocytes, as well as cells from lung, brain, mammary, endothelial, and hematopoietic origins. Further, proto-oncogene tyrosine-protein kinase (c-Src), ERK kinase (MEK), and epidermal growth factor receptors are involved in CAG-induced ERK phosphorylation. CAG may activate telomerase and other cellular effects by activating the Src/MEK/ERK pathway (8). Our previous research showed that CAG induces expression of Janus kinase 2 (JAK2) and signal transducer and activator of transcription 5b (STAT5b) and enhances phosphorylation of STAT5b following an increase in TERT expression. Further, expression of molecules downstream from the JAK/STAT signaling pathway was also increased, suggesting that CAG activates telomerase through the JAK/STAT signaling pathway (9). In addition, CAG-mediated activation of telomerase is associated with the cAMP response element-binding protein (CREB) (3). The above preliminary studies of the mechanisms associated with CAG demonstrate that it activates telomerase and is related to the CREB, MAPK, and JAK/STAT pathways; however, further investigation is needed to comprehensively understand the mechanisms.

Telomerase activation and anti-immunosenescence. A prevailing view is that the immune system gradually ages during the process of senescence. Weak immune systems lead to an increased susceptibility to infections, which accelerates the process of senescence. The immune system consists of immune organs, immune cells, and active immune substances and is associated with senescence (10). Immune cells include T lymphocytes, B lymphocytes, and phagocytes, in which the number of lymphocytes is related to immune function. CAG enhanced both CD8(+) T lymphocytes and CD4(+) T lymphocytes, telomerase activity, and proliferative capacity in vitro $(11,12)$. CD8(+) T lymphocytes from human immunodeficiency virus (HIV)-infected human donors treated with CAG showed an increase in telomerase activity, modest retardation of telomere attrition, increased proliferative potential of $\mathrm{CD} 8(+) \mathrm{T}$ lymphocytes, and enhanced cytokine/chemokine production, thus enhancing the antiviral potential. CAG-induced increases in telomerase activity are blocked by MAKP and ERK inhibitors; therefore, it is likely that CAG-associated enhanced antiviral functions are mediated through the ERK/MAPK pathway (13). In addition to T lymphocyte proliferation in HIV-infected human donors, CAG enhances normal human T lymphocyte proliferation.

An Astragalus membranaceus root extract (HTA; not a single purified compound) has also been shown to be a telomerase activator. However, CAG is a more effective telomerase activator, increasing telomerase activity and the proliferative potential of human CD4 and CD8 T cells more than that of HTA. Further, CAG has been shown to enhance the anti-viral ability of cells and has the potential to ameliorate acquired immunodeficiency syndrome (AIDS); however, the anti-viral properties of CAG have not yet been proven in animal models.

Telomerase activation and anti-pulmonary fibrosis. Within the past decade, numerous diseases or maladies have been associated with mutations in telomerase and/or are known to accelerate telomere loss, including AIDS, congenital dyskeratosis, aplastic anemia, and idiopathic pulmonary fibrosis (IPF). CAG, a telomerase activator, has the possibility to treat these diseases or maladies; to this end, several studies have been conducted. CAG dose-dependently hindered bleomycin-induced fibrosis in mTERT heterozygous mice (TRET+/-mice). However, CAG did not alter the inflammatory response to bleomycin-induced fibrosis, suggesting that the underlying mechanisms are not related to the anti-inflammatory properties of CAG. Prevention against 


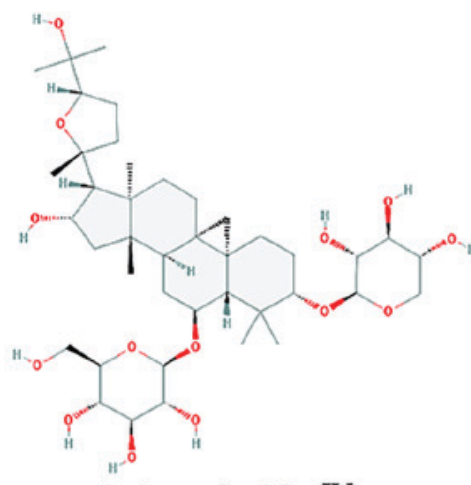

Astragaloside IV

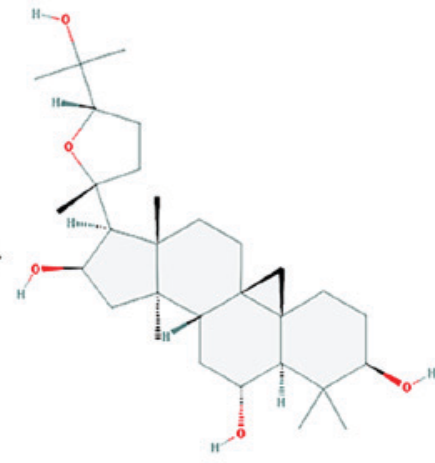

Cycloastragenol

Figure 1. A schematic for cycloastragenol synthesis.

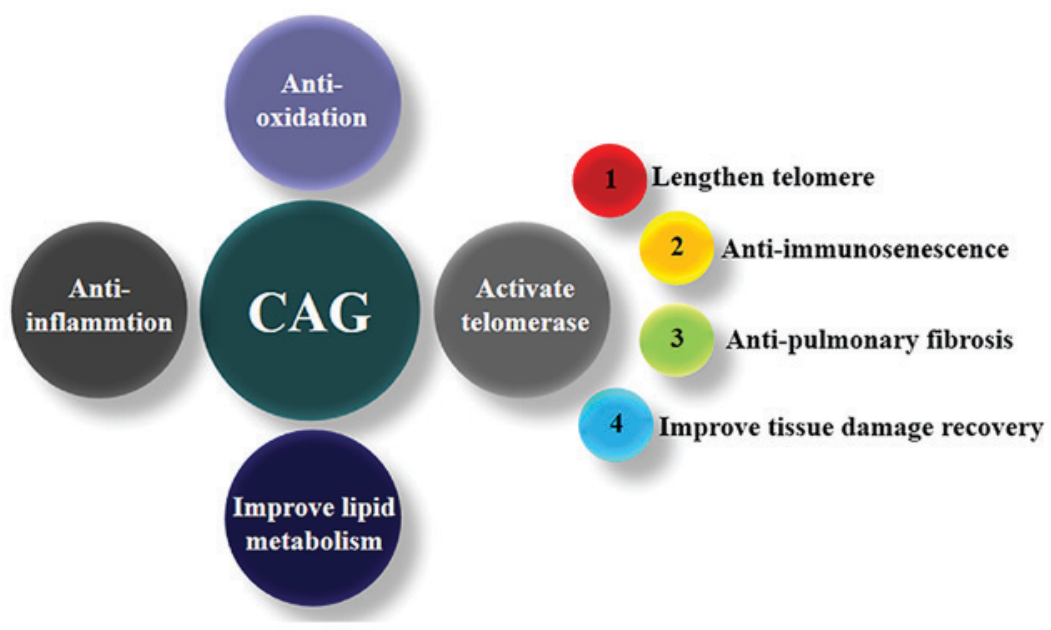

Figure 2. Pharmacological effects of CAG. CAG, cycloastragenol.

fibrosis and suppression of senescence in cells are dependent on telomerase activation. Further, data suggest that the mechanisms associated with the protective effects of CAG against bleomycin-induced lung fibrosis involve specific types of lung cells rather than all cell types in the lung (7). The mechanisms by which CAG works in specific cell types are likely related to the basic cellular proliferative ability; however, this issue has yet to be resolved.

Telomerase activation and recovery of damaged tissue. Telomere length is proportional to cell proliferation at the cellular level; that is, telomerase activation and elongation of telomeres can increase the proliferative ability of cells. The present study indicates that this hypothesis is correct. CAG can improve skin fitness, wound healing, and fur recovery in elderly mice and is a more remarkable agent for wound healing in both in vitro and in vivo assays than that of the other three main saponins from Astragalus species, including AG, cyclocephaloside I (CCI), and cyclocanthoside E (CCE). Of these compounds, CAG is the most effective at inducing human keratinocyte proliferation and migration at a concentration of $1 \mathrm{ng} / \mathrm{ml}$. Further, a 5\% preparation of CAG is the most effective for in vivo wound healing (14). CAG stimulates telomerase activity in human keratinocytes, which may contribute to the wound healing effects associated with CAG.
In addition to its wound healing properties, $\mathrm{CAG}$ promotes recovery from brain injuries, as shown in both cell and animal experiments. Neural stem cells are important for recovery from brain injuries such as hypoxic-ischemic brain injury. CAG increases proliferation and improves survival in neural stem cells and counters the effects of oxygen-glucose deprivation injury in vitro (15). Further, CAG reduces neuron apoptosis, improves nerve functional scoring, and reduces cerebral infarction volume in a cerebral ischemia reperfusion injury rat model in vivo. These functions are related to increasing expression of TERT $(16,17)$. Furthermore, CAG promotes the renewal capacity of flight feathers in captive zebra finches, which are nonmammalian animals (18). In addition, CAG attenuates depression-like behavior in experimental mice. Telomere elongation and activation of telomerase activity are proportional to the tissue telomere-dependent recovery of cells and are potential treatment targets and indicators of tissue recovery.

Improved lipid metabolism. Lipids store energy; however, when lipids accumulate too much, it is harmful for health. CAG could ameliorate various biomarkers associated with lipid metabolism. CAG has been shown to reduce cytoplasmic lipid droplets in 3T3-L1 adipocytes at a low dose. At high doses, CAG prevents differentiation of 3T3-L1 preadipocytes. 
Further, it was observed that CAG dose-dependently stimulates calcium influx in 3T3-L1 preadipocytes. Since elevated intracellular calcium plays a vital role in suppressing adipocyte differentiation, CAG may mediate the balance in lipid metabolism by activating calcium influx in 3T3-L1 preadipocytes (19).

Farnesoid X receptor (FXR) is a potential drug target for the treatment of non-alcoholic fatty liver disease (NAFLD), and CAG is a direct activator of FXR. Animal studies have shown that CAG reduces high-fat diet-induced lipid accumulation in the liver, accompanied by lowered blood glucose levels, serum triglyceride levels, and hepatic bile acids. CAG also ameliorates hepatic steatosis in methionine- and choline-deficient L-amino acid diet (MCD)-induced non-alcoholic steatohepatitis (NASH) mice. These results indicate that CAG ameliorates NAFLD via enhancement of the FXR signaling pathway (20). In addition, CAG increases TERT, c-Myc, and c-Jun expression in the liver and ameliorates liver lipid accumulation as well as glucose tolerance in 2-year old mice. CAG-ameliorated lipid metabolism may occur via multiple mechanisms, and CAG may be a multi-target monomer that binds with various receptors and exerts a variety of functions.

Anti-oxidative properties. The oxidative stress hypothesis is a generally accepted mechanism of senescence. Reactive oxygen species (ROS), including superoxide $\left(\mathrm{O}^{2-}\right)$, hydrogen peroxide $\left(\mathrm{H}_{2} \mathrm{O}_{2}\right)$, and the hydroxyl radical $(-\mathrm{OH})$ damage cell functions and are a primary cause of disease and cell senescence. In a D-galactose-induced senescence mouse model, CAG treatment up-regulated total antioxidant capacity (T-AOC) and superoxide dismutase (T-SOD) activity, increased hydroxyproline (HYP) stores, and down-regulated malondialdehyde (MDA) in the skin, heart, and liver. Further, CAG enhanced the antioxidant capacity in a non-dose-dependent manner (21). The anti-oxidant effects of CAG may be related to the hydroxyl found in the chemical structure of CAG. Since oxidative stress is a main reason for telomere attrition (22), the telomere-protective effects of CAG may be related to both its antioxidant and telomerase activation properties.

Anti-inflammatory properties. Inflammation is a very common and important basic pathological process. Body surface trauma, infections, common organ diseases, and frequently-occurring diseases (such as pneumonia, hepatitis, and nephritis) are all related to inflammation. ROS generation was suppressed in human umbilical vein EA.hy926 cells exposed to CAG in the setting of endoplasmic reticulum (ER) stress. Further, CAG attenuates phosphorylation of inositol-requiring enzyme 1 (IRE1)- $\alpha$, suppresses thioredoxin-interacting protein/NLR family pyrin domain containing 3 (TXNIP/NLRP3) inflammasome activation, inhibits mitochondrial cell death, and reduces apoptosis in endothelial cells. Further, CAG enhances 5'AMP-activated protein kinase (AMPK) phosphorylation, an effect that is diminished by AMPK inhibitors, indicating a potential role for AMPK in the anti-inflammatory properties of CAG (23).

We discussed the role of CAG in the normal immune system above; however, CAG also plays a role in the activated immune system. The effects of CAG in a concanavalin (Con) A pan-activated lymphocyte model were reported by
Sun et al (24). CD69 (an early-activated marker) and CD25 (a middle-activated marker), both of which are expressed on the surface of lymphocytes, are inhibited in lymphocytes pretreated with CAG and are activated via Con A treatment in vitro. Further, pretreatment with $\mathrm{CAG}$ blocks Con $\mathrm{A}$-induced lymphocyte mitogenesis, induces cell-cycle arrest in the G0/G1-phase, and downregulates cytokine expression. In addition, $\mathrm{Ca}^{2+}$ release from intracellular ER storage and $\mathrm{Ca}^{2+}$ influx from the extracellular environment are inhibited in Con A-induced activated T-cells treated with CAG; these changes may be related to the anti-inflammatory properties of CAG (24). CAG could activate the immune system and inhibit activated immunity similar to that of feedback inhibition. It is possible that the structure of CAG resembles substances found in our body, and it exerts its functions in a similar way.

Efficacy base on clinical trial. The Patton protocol-1 provided 37 subjects with a comprehensive dietary supplement pack containing CAG for 12 months. This clinical trial concluded that CAG lengthens critically short telomeres and remodels the relative proportion of circulating leukocytes in cytomegalovirus-positive $[\mathrm{CMV}(+)]$ subjects toward a more 'youthful' profile, as seen in CMV(-) subjects (25). Furthermore, the supplement pack improved biomarkers of metabolic, bone, and cardiovascular health, such as fasting glucose, insulin, cholesterol, blood pressure, and bone mineral density (BMD). These effects are mostly attributable to CAG (26). A study reported by Harley et al (26), reported an increase in BMD in naturally aging rats; clinical trials have further verified this function. It is noteworthy that the Patton protocol-1 did not establish a control before initiating the study, which may have affected the trial results.

A random clinical trial conducted in 117 relatively healthy CMV (+) subjects 53-87 years old verified that TA-65 can significantly lengthen telomeres (4). Other TCM extracts, such as Cynomorium songaricum polysaccharides and Epimedium brevicornu flavonoids, can also activate telomerase or protect telomeres; however, CAG is the only compound that has been shown to activate telomerase in humans to date. Further, a randomized placebo-controlled study showed that CAG significantly improves the macular function of treatment subjects (27).

CAG activates human telomerase and has various positive functions. It is still unknown whether these different functions are related to each other and to CAG dosages and whether CAG functions differently in different groups of people.

\section{Adverse reactions}

Critically short telomeres will lead to cell senescence and apoptosis, and numerous diseases or maladies are related to short telomeres. Telomerase activators could attenuate this process. However, telomeres are elongated via the telomerase enzyme in more than $80 \%$ of tumors. As such, activated telomerase is a hallmark of cancer (28). As CAG is a telomerase activator, it is vital to understand the adverse reactions associated with CAG treatment regimens. No treatment-related mortalities were observed in rats ingesting $0,40,80$, or $150 \mathrm{mg} / \mathrm{kg} /$ day CAG via oral gavage for 91 consecutive days in a subchronic study with a 4-week recovery period; further, 
no adverse effects were observed. In male and female rats, the oral no-observed-adverse-effect level (NOAEL) of CAG was $>150 \mathrm{mg} / \mathrm{kg} /$ day. In addition, CAG did not induce any toxic or genotoxic effect according to the result of bacterial reverse mutation assay, in vitro chromosome aberration assay and in vivo erythrocyte micronucleus assay (29). Administration of CAG for 4 months in adult female mice $(25 \mathrm{mg} / \mathrm{kg} /$ day $) \mathrm{did}$ not increase the incidence of cancer. However, CAG competitively inhibited UDP-glucuronosyltransferase (UGT) 1A8 and noncompetitively inhibited UGT2B7. Herb-drug interactions between CAG and drugs that are mainly metabolized by UGT1A8 or UGT2B7 might occur in vivo when the plasma concentration of CAG is above 0.034 and $20.98 \mu \mathrm{M}$, respectively (30). Furthermore, the Patton protocol-1, a commercial health maintenance program that provided 7000 person-years of use with a comprehensive dietary supplement pack containing CAG over 5 years, reported no adverse events or effects attributed to administration of the dietary supplement pack (26).

According to current research results, CAG is relatively safe within a certain dose range and has no serious adverse reactions; CAG was determined to be generally recognized as safe (GRAS) by an independent expert panel of the Food and Drug Administration (FDA) on the 19th of November, 2014. CAG has been used as a medical food (31). However, telomerase activation has been observed in $80 \%$ of human tumors, suggesting that telomerase plays a nonnegligible role in tumor development (32). Thus, the potential adverse reactions of CAG should be noted. More studies regarding adverse reactions of CAG are necessary to fully understand how to use CAG rationally.

\section{Pharmacokinetics}

CAG is efficiently absorbed through the intestinal epithelium by passive diffusion, as shown by a study that investigated the intestinal absorption and metabolism of CAG using an in vitro models composed of human small intestinal mucosa (Caco-2 model) and liver microsomes. However, metabolites were found in the apical and basolateral sides of Caco-2 monolayers, suggesting that first-pass intestinal metabolism of CAG might occur upon passage through the intestinal epithelium. After incubation for $30 \mathrm{~min}, \mathrm{CAG}$ underwent extensive metabolism in rat (17.4\%) and human (8.2\%) liver microsomes (33). An comparison of CAG metabolizing ability of different rat tissues showed that the metabolic capacity of heart, spleen, lung, and kidney was lower than that of the liver in vivo (34). Further, a significant inhibitory effect on the cytochrome $\mathrm{P}_{450}$ 3A4 (CYP3A4) subunit, as well as significant induction of the CYP2E1 subunit were observed after administration of CAG via continuous gavage (35). These results indicate that although CAG is efficiently absorbed through the intestinal epithelium, its oral bioavailability CAG would be limited by extensive first-pass hepatic metabolism.

\section{Discussion}

$C A G$ naming. In the process of searching documents, we found that CAG (CAS number: 78574-94-4) is noted by diverse names in different documents. CAG is named
(2aR,3R,4S,5aS,5bS,7S,7aR,9S,11aR,12aS)-3-((2R,5S)-5(2-hydroxypropan-2-yl)-2-methyltetrahydrofuran-2-yl)-2a,5 a,8,8-tetramethylhexadecahydrocyclopenta[a]cyclopropa[e] phenanthrene-4,7,9-triol, other synonyms include CAG, CA, astramembrangenin, cyclogalegigenin, GRN510, and TA-65. TA- 65 was named by the T.A Science Corporation as a nutrition and health care product. A document reported that GRN510 is a novel and proprietary chemical entity derived from GRN665/TAT2. However, GRN510 is considered equivalent to CAG in PubChem (an open chemistry database); therefore, we considered GRN510 as equivalent to CAG in this article. For simplicity, we termed cycloastragenol as CAG in this report.

Advantages and disadvantages associated with CAG. CAG has multiple pharmacological effects, including activation of telomerase, improved lipid metabolism, anti-oxidation, and anti-inflammatory properties. Clinical trials have proven components involved in CAG functions. No serious adverse effects are associated with CAG within a certain dose range. However, the carcinogenic potential of CAG is the main factor preventing the use of CAG clinically. Clarification of the quantitative effects and efficacy associated with CAG in different individuals is needed.

Unknown information regarding $C A G$. While there is a large body of literature regarding $\mathrm{CAG}$, there are still significant gaps in knowledge.

CAG stimulates calcium influx or inhibits calcium influx depending on the cell type and pathological state. Further, CAG has different pharmacological effects at diverse concentrations in 3T3-L1 preadipocytes. The underlying mechanisms regarding these effects have not yet been reported.

CAG has extensive pharmacological effects; however, the detailed underlying mechanisms for most of these effects rem ain unclear. A summary of CAG-associated effects and the corresponding mechanisms are show in Table I. Mechanisms underlying the extensive pharmacological effects associated with CAG are complex. We attempted to establish a hypothetical mechanism for the effects of CAG based on previous results (Fig. 3).

CAG could increase BMD and improve glucose metabolism in naturally aged rats. There is no literature describing whether CAG has anti-osteoporosis or anti-diabetic effects in corresponding animal models.

\section{Conclusions and prospective}

Conclusions. In this review, the current state of CAG research is detailed and elucidated, and the efficacy, pharmacokinetics, and adverse reactions of CAG are summarized. According to the present research results, CAG has extensive pharmacological effects, including telomerase activation, telomere elongation, anti-inflammation, anti-oxidation, anti-viral, anti-pulmonary fibrosis, anti-ischemic and hypoxic injury, and anti-lipid accumulation properties. Studies also suggest that CAG improves liver metabolic homeostasis, promotes wound healing, promotes feather growth, and improves certain health-span indicators both in humans and animals. However, more attention and further studies are needed to evaluate whether CAG has potential adverse reactions, and studies examining the detailed 


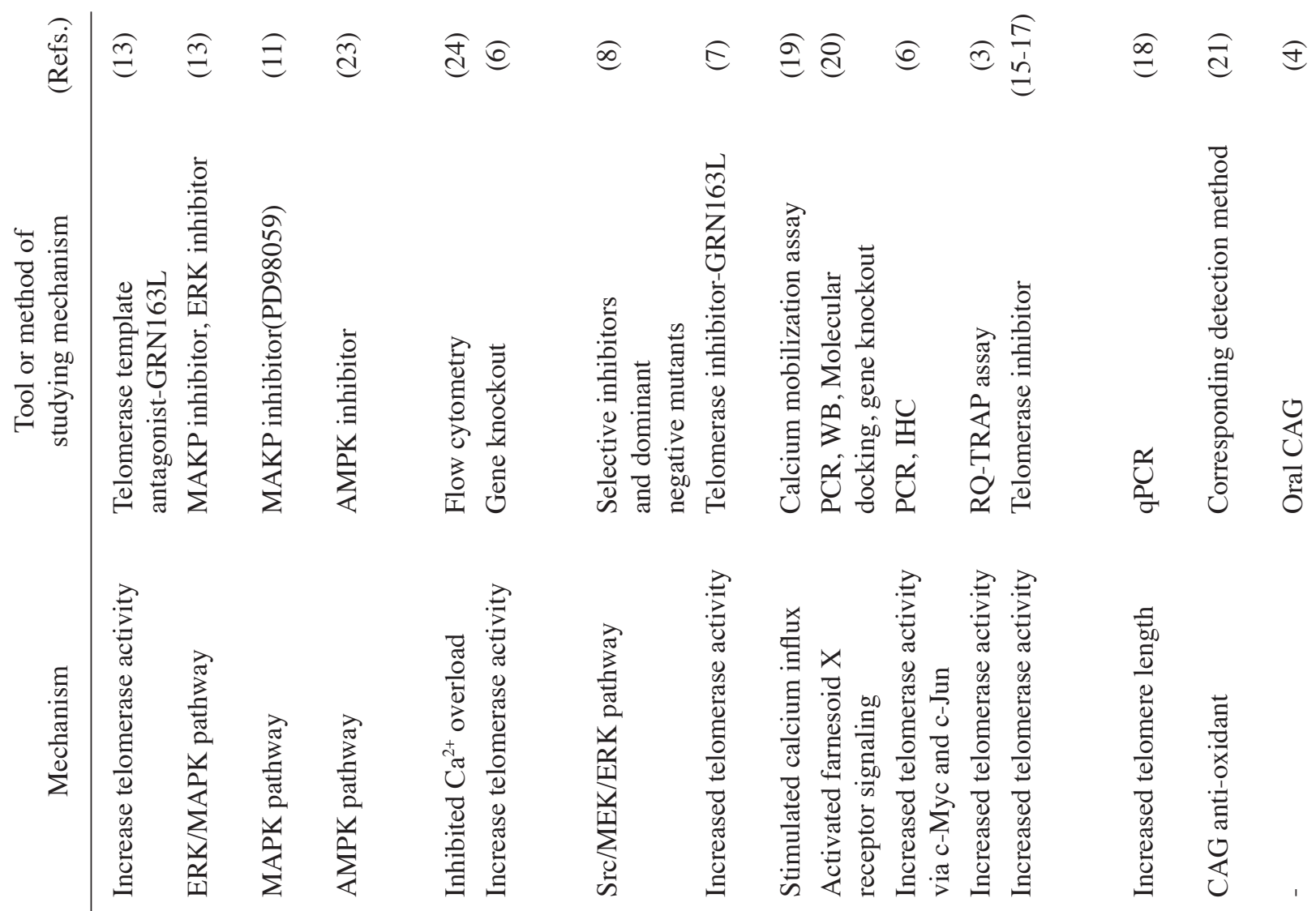

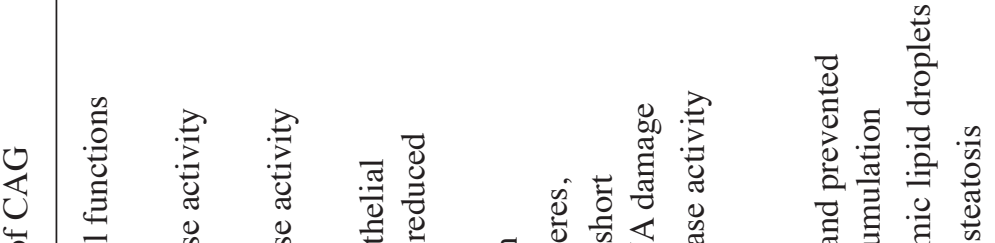
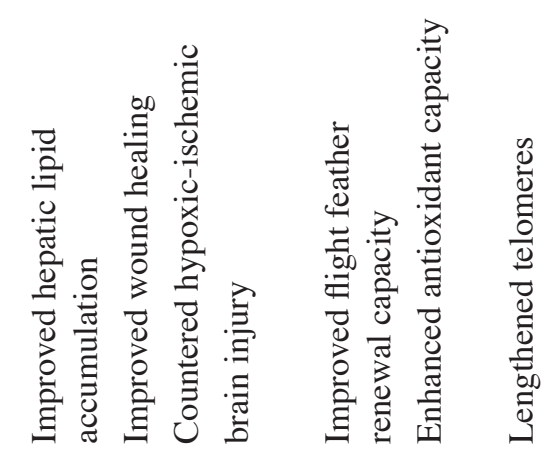

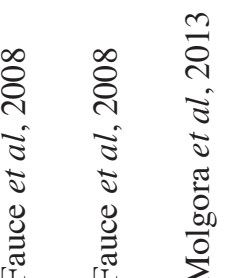

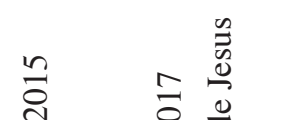

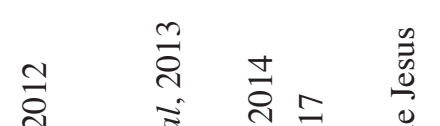
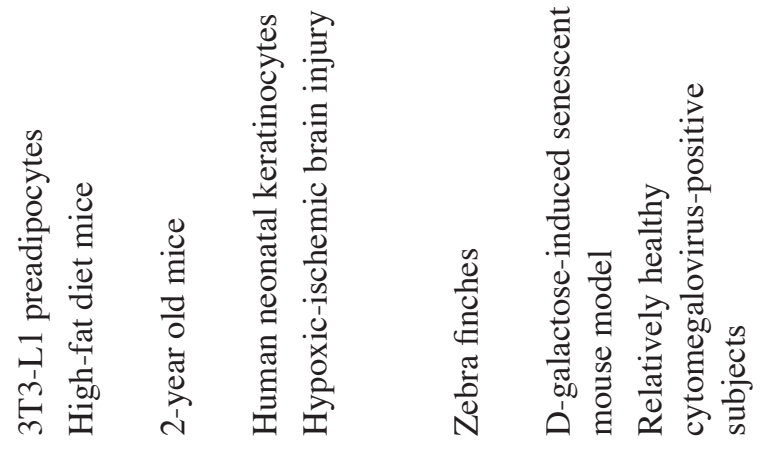

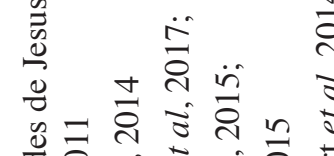

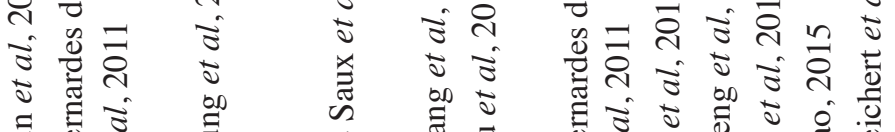

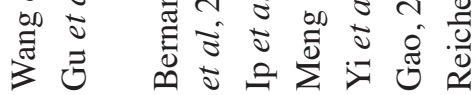

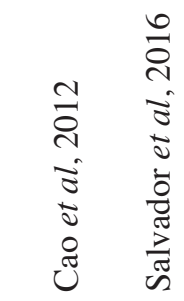




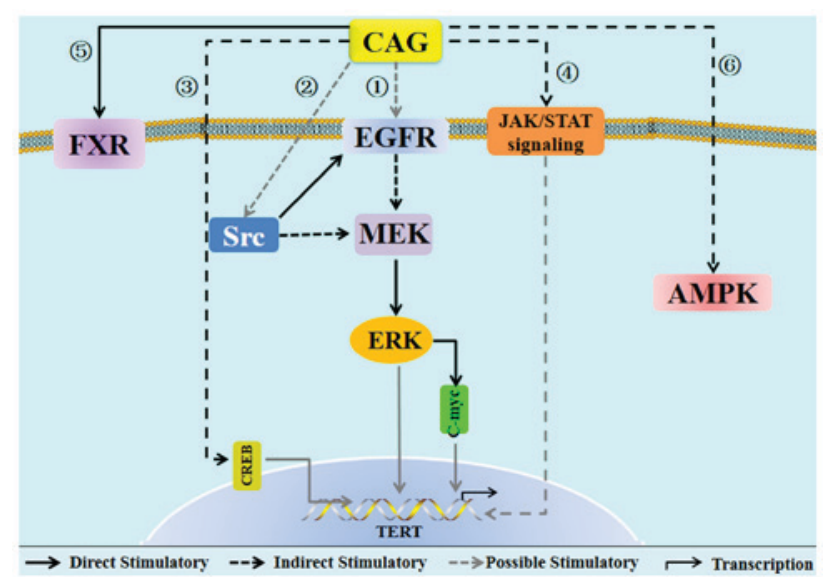

Figure 3. Hypothetical mechanisms associated with CAG. Arrow heads represent stimulatory modifications. Solid lines are direct stimulatory modifications based on the literature. Dashed lines indicate stimulatory modifications; grey dashed lines indicate possible stimulatory modifications. CAG may activate telomerase through pathway $1,2,3$ and 4 , and then exert various effects. Through pathway 5, CAG directly stimulates the FXR to improve hepatitis. Through pathway 6, CAG indirectly stimulates AMPK to improve inflammation. CAG, cycloastragenol; FXR, farnesoid X receptor; AMPK, 5'adenosine monophosphate-activated protein kinase; EGFR, epidermal growth factor receptor; ERK, extracellular signal-regulated kinase; MEK, mitogen-activated protein kinase kinase; JAK, Janus kinase; STAT, signal transducer and activator of transcription; TERT, telomerase reverse transcriptase.

underlying mechanisms associated with CAG are required. In addition, further multicenter clinical studies are required for specific diseases such as steatohepatitis, AIDS, pulmonary fibroses, wound healing etc.

Prospective. We anticipate that researchers will conduct more studies on CAG to evaluate its efficacy and adverse reactions, as well as understand how to use CAG rationally to enhance its effects and reduce adverse reactions so that $\mathrm{CAG}$ can be used for the clinical treatment of corresponding diseases. We believe that development of these clinical uses will benefit people, which is the purpose of this article.

\section{Acknowledgements}

Not applicable.

\section{Funding}

The present study was supported by grants from National Natural Science Foundation of China (grant nos. 81102450 and 81673814), the Open Fund Project of Key Laboratory of Guangdong Province (grant no. 4CX16010G), the Science and Technology Plan of Guangdong Province (grant nos. 2016ZC0178 and 2016A020215148) and Open Foundation of Guangdong Key Laboratory for Research and Development of Natural Drugs (grant no. TRYW201603).

\section{Availability of data and materials}

The datasets used and/or analyzed during the current study are available from the corresponding author on reasonable request. 


\section{Authors' contributions}

LZ performed the literature review and mapping. YL and YYg made suggestions and edited the manuscript. YYu wrote the manuscript.

\section{Ethics approval and consent to participate}

Not applicable.

\section{Patient consent for publication}

Not applicable.

\section{Competing interests}

The authors declare that they have no competing interests.

\section{References}

1. Shen CY, Jiang JG, Yang L, Wang DW and Zhu W: Anti-ageing active ingredients from herbs and nutraceuticals used in traditional Chinese medicine: Pharmacological mechanisms and implications for drug discovery. Br J Pharmacol 174: 1395-1425, 2017.

2. Zhang S, Lu J, Yan J and Zhang H: Research progress of preparation technology and pharmacological effect of cycloastragenol Chin J New Drugs 16: 1872-1875, 2016.

3. Ip FC, Ng YP, An HJ, Dai Y, Pang HH, Hu YQ, Chin AC, Harley CB, Wong YH and Ip NY: Cycloastragenol is a potent telomerase activator in neuronal cells: Implications for depression management. Neurosignals 22: 52-63, 2014.

4. Salvador L, Singaravelu G, Harley CB, Flom P, Suram A and Raffaele JM: A natural product telomerase activator lengthens telomeres in humans: A randomized, double blind and placebo controlled study. Rejuvenation Res 19: 478-484, 2016.

5. Hemann MT, Strong MA, Hao LY and Greider CW: The shortest telomere, not average telomere length, is critical for cell viability and chromosome stability. Cell 107: 67-77, 2001.

6. Bernardes de Jesus B, Schneeberger K, Vera E, Tejera A, Harley CB and Blasco MA: The telomerase activator TA-65 elongates short telomeres and increases health span of adult/old mice without increasing cancer incidence. Aging Cell 10: 604-621, 2011.

7. Le Saux CJ, Davy P, Brampton C, Ahuja SS, Fauce S, Shivshankar P, Nguyen H, Ramaseshan M, Tressler R, Pirot Z, et al: A novel telomerase activator suppresses lung damage in a murine model of idiopathic pulmonary fibrosis PLoS One 8: e58423, 2013.

8. Yung LY, Lam WS, Ho MK, Hu Y, Ip FC, Pang H, Chin AC, Harley CB, Ip NY and Wong YH: Astragaloside IV and cycloastragenol stimulate the phosphorylation of extracellular signal-regulated protein kinase in multiple cell types. Planta Med 78: 115-121, 2012.

9. Li J: Astragaloside IV and cycloastragenol attenuate osteoblastic injury and aging elicited by oxidative stress, 2017.

10. Bektas A, Schurman SH, Sen R and Ferrucci L: Human T cell immunosenescence and inflammation in aging. J Leukoc Biol 102: 977-988, 2017.

11. Molgora B, Bateman R, Sweeney G, Finger D, Dimler T, Effros RB and Valenzuela HF: Functional assessment of pharmacological telomerase activators in human $\mathrm{T}$ cells. Cells 2 : 57-66, 2013.

12. Verotta L, Guerrini M, El-Sebakhy NA, Assad AM, Toaima SM, Radwan MM, Luo YD and Pezzuto JM: Cycloartane and oleanane saponins from egyptian Astragalus spp. as modulators of lymphocyte proliferation. Planta Med 68: 986-994, 2002.

13. Fauce SR, Jamieson BD, Chin AC, Mitsuyasu RT, Parish ST, Ng HL, Kitchen CM, Yang OO,Harley CB and Effros RB: Telomerase-based pharmacologic enhancement of antiviral function of human CD8+ T lymphocytes. J Immunol 181: 7400-7406, 2008.

14. Sevimli-Gur C, Onbasilar I, Atilla P, Genç R, Cakar N, Deliloğlu-Gürhan I and Bedir E: In vitro growth stimulatory and in vivo wound healing studies on cycloartane-type saponins of Astragalus genus. J Ethnopharmacol 134: 844-850, 2011.
15. Meng JJ, Li SP, Zhao FY, Tong Y, Mu DZ and Qu Y: Effect of telomerase activation on biological behavior of neural stem cells in hypoxic-ischemic rats. Zhongguo Dang Dai Er Ke Za Zhi 19: 229-36, 2017 (In Chinese).

16. Yi Z and Gao W: Protective effects of cycloastragenol on focal cerebral ischemia reperfusion injury in rats. Journa Cheng Medical Collage: 186-188, 2015.

17. Gao W: Pfect of cycloastragenol on neuronal apoptosis and TERT expression after cerebral ischemia reperfusion in rats. Chin J Pathophysiology 10: 1858, 2015.

18. Reichert S, Bize P, Arrive M, Zahn S, Massemin S and Criscuolo F: Experimental increase in telomere length leads to faster feather regeneration. Exp Gerontol 52: 36-38, 2014.

19. Wang S, Zhai C, Liu Q, Wang X, Ren Z, Zhang Y, Zhang Y, Wu Q, Sun S, Li S and Qiao Y: Cycloastragenol, a triterpene aglycone derived from Radix astragali, suppresses the accumulation of cytoplasmic lipid droplet in 3T3-L1 adipocytes. Biochem Biophysical Res Commun 450: 306-311, 2014.

20. Gu M, Zhang S, Zhao Y, Huang J, Wang Y, Li Y, Fan S, Yang L, Ji G, Tong Q and Huang C: Cycloastragenol improves hepatic steatosis by activating farnesoid $\mathrm{X}$ receptor signalling. Pharmacol Res 121: 22-32, 2017.

21. Cao Y, Li W, Wei L, Liu X, Li Z and He W: Anti-aging effect of cyclogreitol on D-galactose induced aging mice. Chin J Exp Tradit Med Formulae: 208-211, 2012.

22. Richter T and von Zglinicki T: A continuous correlation between oxidative stress and telomere shortening in fibroblasts. Exp Gerontol 42: 1039-1042, 2007.

23. Zhao Y, Li Q, Zhao W, Li J, Sun Y, Liu K, Liu B and Zhang N: Astragaloside IV and cycloastragenol are equally effective in inhibition of endoplasmic reticulum stress-associated TXNIP/NLRP3 inflammasome activation in the endothelium. J Ethnopharmacol 169: 210-218, 2015.

24. Sun C, Jiang M, Zhang L, Yang J, Zhang G, Du B, Ren Y, Li X and Yao J: Cycloastragenol mediates activation and proliferation suppression in concanavalin A-induced mouse lymphocyte pan-activation model. Immunopharmacol Immunotoxicol 39: 131-139, 2017.

25. Harley CB, Liu W, Blasco M, Vera E, Andrews WH, Briggs LA and Raffaele JM: A natural product telomerase activator as part of a health maintenance program. Rejuvenation Res 14: 45-56, 2011.

26. Harley CB, Liu W, Flom PL and Raffaele JM: A natural product telomerase activator as part of a health maintenance program: metabolic and cardiovascular response. Rejuvenation Res 16: 386-395, 2013.

27. Dow CT and Harley CB: Evaluation of an oral telomerase activator for early age-related macular degeneration-a pilot study. Clin Ophthalmol 10: 243-249, 2016.

28. Aschacher T, Wolf B, Enzmann F, Kienzl P, Messner B, Sampl S, Svoboda M, Mechtcheriakova D, Holzmann K and Bergmann M: LINE-1 induces hTERT and ensures telomere maintenance in tumour cell lines. Oncogene 35: 94-104, 2016.

29. Szabo NJ: Dietary safety of cycloastragenol from Astragalus spp: Subchronic toxicity and genotoxicity studies. Food Chem Toxicol 64: 322-334, 2014.

30. Ran R, Zhang C, Li R, Chen B, Zhang W, Zhao Z, Fu Z, Du Z, Du X, Yang X and Fang Z: Evaluation and comparison of the inhibition effect of astragaloside IV and aglycone cycloastragenol on various UDP-Glucuronosyltransferase (UGT) isoforms. Molecules 21: E1616, 2016.

31. T.A. Sciences ${ }^{\circledR}$ : TA- $65^{\circledR}$ Receives Generally Recognized as Safe Status. https://www.tasciences.com/ta-65-receives-generallyrecognized-safe-status/. Accessed November 19, 2014.

32. Shay JW and Bacchetti S: A survey of telomerase activity in human cancer. Eur J Cancer 33: 787-791, 1997.

33. Zhu J, Lee S, Ho MK, Hu Y, Pang H, Ip FC, Chin AC, Harley CB, Ip NY and Wong YH: In vitro intestinal absorption and first-pass intestinal and hepatic metabolism of cycloastragenol, a potent small molecule telomerase activator. Drug Metab Pharmacokinet 25: 477-486, 2010.

34. Liu X: In vitro and in vivo metabolism research of astragaloside iv and cycloastragenol (unpublished $\mathrm{PhD}$ thesis). Beijing University of Chinese Medicine, 2013.

35. Wei B, Ye J, Bi B, Sun G, Yang X and Zhang Y: Effect of continuous intragastric administration of cycloastragenol on liver $\mathrm{p} 450$ enzymes in rats. Chin J New Drugs: 476-479, 2014.

This work is licensed under a Creative Commons Attribution-NonCommercial-NoDerivatives 4.0 International (CC BY-NC-ND 4.0) License. 